\title{
The value of forceps biopsy and core needle biopsy in prediction of pathologic complete remission in locally advanced rectal cancer treated with neoadjuvant chemoradiotherapy
}

\author{
Jing-Hua Tang ${ }^{1,2, *}$, Xin An ${ }^{1,3, *}, X_{i}$ Lin $^{1,4, *}$, Yuan-Hong Gao ${ }^{1,5}$, Guo-Chen Liu ${ }^{1,2}$, Ling-Heng \\ Kong $^{1,2}$, Zhi-Zhong Pan ${ }^{1,2}$, Pei-Rong Ding ${ }^{1,2}$ \\ ${ }^{1}$ State Key Laboratory of Oncology in South China, Collaborative Innovation Center for Cancer Medicine, Guangzhou, China \\ ${ }^{2}$ Departments of Colorectal Surgery, Sun Yat-sen University Cancer Center, Guangzhou, China \\ ${ }^{3}$ Departments of Medical Oncology, Sun Yat-sen University Cancer Center, Guangzhou, China \\ ${ }^{4}$ Departments of Ultrasound, Sun Yat-sen University Cancer Center, Guangzhou, China \\ ${ }^{5}$ Departments of Radiation Oncology, Sun Yat-sen University Cancer Center, Guangzhou, China \\ *These authors have contributed equally to this work \\ Correspondence to: \\ Pei-Rong Ding, e-mail: dingpr@mail.sysu.edu.cn \\ Zhi-Zhong Pan, e-mail: panzhzh@sysucc.org.cn
}

Keywords: forceps biopsy, core needle biopsy, locally advanced rectal cancer, neoadjuvant chemoradiotherapy

Received: June 22, 2015

Accepted: September 08, 2015

Published: September 18, 2015

\section{ABSTRACT}

Patients with pathological complete remission (PCR) after treated with neoadjuvant chemoradiotherapy (nCRT) have better long-term outcome and may receive conservative treatments in locally advanced rectal cancer (LARC). The study aimed to evaluate the value of forceps biopsy and core needle biopsy in prediction of PCR in LARC treated with nCRT. In total, 120patients entered this study. Sixty-one consecutive patients received preoperative forceps biopsy during endoscopic examination. Ex vivo core needle biopsy was performed in resected specimens of another $\mathbf{4 3}$ consecutive patients. The accuracy for ex vivo core needle biopsy was significantly higher than forceps biopsy (76.7\% vs. $36.1 \% ; p<0.001)$. The sensitivity for ex vivo core needle biopsy was significantly lower in good responder (TRG 3) than poor responder (TRG $\leq 2)(52.9 \%$ vs. $94.1 \%$; $p=0.017$ ). In vivo core needle biopsy was further performed in 16 patients with good response. Eleven patients had residual cancer cells in final resected specimens, among whom $4(36.4 \%)$ patients were biopsy positive. In conclusion, routine forceps biopsy was of limited value in identifying PCR after nCRT. Although core needle biopsy might further identify a subset of patients with residual cancer cells, the accuracy was not substantially increased in good responders.

\section{INTRODUCTION}

Neoadjuvant chemoradiotherapy (nCRT) followed by total mesorectal excision (TEM) and systemic chemotherapy is the standard treatment for patients with locally advanced rectal cancer (LARC) [1]. With this approach, $15-36 \%$ of patients have no residual viable cancer cells at pathological examination, referred as pathological complete remission (pCR), and these patients have better long-term outcome $[2,3]$. Studies suggest that conservative approaches, such as local excision or even avoidance of surgery, might be alternative choices without compromising the oncologic outcome for patients with $\mathrm{pCR}[4,5]$.
To date, there are still no reliable methods to identify patients with pCR before radical surgery. As a result, clinical complete response (cCR), defined as no clinical detectable tumor by physical examination, endoscopic evaluation, and imaging, is designed as a surrogate endpoint for pCR [4, 6]. However, the concordance between cCR and pCR varies from $22 \%$ to $96 \%$ in different reports [7-10], which questions the clinical value of such strategies. Therefore, it is of crucial significance to develop novel strategy to predict $\mathrm{pCR}$ for patients after nCRT in LARC.

Forceps biopsy and core needle biopsy are frequently used for the diagnosis of malignant tumors. 
Core needle biopsy is considered to be more accurate especially in tumor beneath epithelium which is a common phenomenon in LARC after neoadjuvant CRT. So far, there is no study reporting its value in evaluating $\mathrm{pCR}$ in the patients treated with neoadjuvant CRT. This study aimed to evaluate the value of forceps biopsy and core needle biopsy in predicting pCR in LARC after nCRT.

\section{RESULTS}

\section{Patients' demographics}

Sixty-one patients received endoscopic forceps biopsy, among whom 12 (19.7\%) patients achieved pCR. Ex vivo core needle biopsy was performed in 43 patients, among whom $8(18.6 \%)$ achieved pCR. No statistically significant differences in the baseline characteristics were found between patients undergoing forceps biopsy and ex vivo core needle biopsy, except the interval between radiotherapy (RT) completion and biopsy (Table 1). In vivo core needle biopsy was performed in 16 patients with good response after CRT, and $5(31.3 \%)$ patients achieved pCR (Table 2$)$. There was no overlapping between different groups.

\section{Diagnostic performance of endoscopic forceps biopsy}

Sixty-one patients received endoscopic forceps biopsy, among whom 51 patients were negative at biopsy and only 12 patients had no viable cancer cells found in the final resected specimens. The negative predictive value referred as the predictive value of pCR was $23.5 \%$. Forty-nine patients had residual cancer cells in the final surgical specimens, among whom 10 patients were biopsy positive. The sensitivity was $20.4 \%$, and the specificity was $100 \%$. The overall accuracy was $36.1 \%$. The result of histopathological findings of forceps biopsy compared with the surgical specimen was listed in Table 3.

Of the 49 patients with residual cancer cells at resected specimen (non-pCR), 16 (32.7\%) patients were $\mathrm{TRG} \leq 2$ and $33(67.3 \%)$ patients were $\mathrm{TRG}=3$. The sensitivity was not significantly different between TRG $\leq$ 2 and $\mathrm{TRG}=3(31.3 \%$ vs. $15.2 \% ; p=0.351)$ (Table 4).

\section{Diagnostic performance of ex vivo core needle biopsy}

Ex vivo core needle biopsy was performed in 43 resected specimens. Of the 18 patients with biopsy negative, 8 achieved pCR. The negative predictive value was $44.4 \%$. Thirty-five patients had residual cancer cells in the final resected specimens, among whom 25 patients were positive at core needle biopsy. The sensitivity was $71.4 \%$, and the specificity was $100 \%$. The overall accuracy was $76.7 \%$. Concordance between surgical specimen and ex vivo core needle biopsy samples was listed in Table 5.
Among 35 patients with residual cancer cells in the final resected specimens, 17 patients were $\mathrm{TRG} \leq 2 ; 17$ patients were $\mathrm{TRG}=3$; and 1 patient was pathological complete regression in primary tumor (ypT0 or TRG4) but had residual tumor cells in the lymph nodes (ypN+). The sensitivity was $94.1 \%$ with $\mathrm{TRG} \leq 2$ and $52.9 \%$ with TRG $=3$. The sensitivity was significantly different between $\mathrm{TRG} \leq 2$ and $\mathrm{TRG}=3(p=0.017)$ (Table 6).

\section{Diagnostic performance of in vivo core needle biopsy}

Sixteen patients with good response evaluated by digital rectal examination, colonoscopy and forceps biopsy, and MRI after nCRT received in vivo core needle biopsy. Residual cancer cells were detected in 11 (68.8\%) patients at the final pathologic examination, among whom 4 patients were biopsy positive. The sensitivity was $36.4 \%$, and the specificity was $100 \%$. Of the 12 patients negative at in vivo core needle biopsy, 5 patients achieved pCR. The negative predictive value was $41.7 \%$. The overall accuracy was 56.3\% (Table 7). Except for some discomfort experienced during the biopsy, no serious procedure-related complications were observed in any of these 16 patients.

\section{DISCUSSION}

Our study demonstrates that forceps biopsy is an inaccurate method of identifying patients with pCR after nCRT. Although the accuracy of ex vivo core needle biopsy has been markedly improved compared to forceps biopsy, the accuracy of in vivo core needle biopsy is far from perfect in the patients with good response. To our knowledge, it is the first study to analyze the value of core needle biopsy in predicting pCR through ex vivo and in vivo. The findings of this study may have significant clinical implications because they highlight the limitation of forceps biopsy and core needle biopsy in prediction of pCR in LARC treated with nCRT.

In patients with negative forceps biopsy, only $23.5 \%$ patients achieved pCR, which is consistent with the previous reports showing that the negative predictive value for forceps biopsy was $21.4 \%$ and $36 \%[11,12]$. Similarly, in a Habr-Gama's study, the negative predictive value was $21 \%$ for those with significant tumor downsizing [13]. The low accuracy might be attributed to the redistribution of the residual cancer cells after CRT. Duldulao MP et al revealed that residual cancer cells in rectal cancer after CRT preferentially located to the invasive front of the tumor, and at least $25 \%$ patients with cT2 tumors and more than $40 \%$ patients with cT3/4 tumors after CRT that did not achieve pCR would have no residual cancer cells in the mucosa or submucosa [14]. Dana M. Hayden and Fraser M. Smith reported that residual tumor cells were presented lateral and distal to the residual mucosal 
Table 1: Demographics of patients according to different biopsy approaches

\begin{tabular}{|c|c|c|c|}
\hline Demographics & $\begin{array}{l}\text { Forceps biopsy } \\
\quad(n=61)\end{array}$ & $\begin{array}{l}\text { Ex vivo core needle biopsy } \\
(n=43)\end{array}$ & $p$ value \\
\hline Age, years & $54(25-74)$ & $55(28-84)$ & 0.747 \\
\hline Gender (female/male) & $16 / 45$ & $18 / 25$ & 0.137 \\
\hline Distance from anal verge, $\mathrm{cm}$ & $6.5(1.0-13.0)$ & $5.3(0.5-12.0)$ & 0.056 \\
\hline $\begin{array}{l}\text { Interval between RT and } \\
\text { biopsy, days }\end{array}$ & $40.25(21-83)$ & $54.5(29-80)$ & $<0.001$ \\
\hline $\begin{array}{l}\text { Interval between RT and } \\
\text { surgery, days }\end{array}$ & $56.9(42-82)$ & $54.5(29-80)$ & 0.287 \\
\hline \multicolumn{4}{|l|}{ Clinical T stage } \\
\hline cT2 & $6(9.8)$ & $3(7.0)$ & 0.809 \\
\hline cT3 & $32(52.5)$ & $26(60.5)$ & \\
\hline cT4 & $23(37.7)$ & $14(32.6)$ & \\
\hline \multicolumn{4}{|l|}{ Clinical N stage } \\
\hline $\mathrm{cN} 0$ & $21(34.4)$ & $16(37.2)$ & 0.836 \\
\hline $\mathrm{cN}+$ & $40(65.6)$ & $27(62.8)$ & \\
\hline \multicolumn{4}{|l|}{ Pathologic T stage } \\
\hline урТ0 & $12(19.7)$ & $9(20.9)$ & 0.701 \\
\hline ypTis & $1(1.6)$ & $1(2.3)$ & \\
\hline урT1 & $2(3.3)$ & $0(0)$ & \\
\hline урT2 & $12(19.7)$ & $5(11.6)$ & \\
\hline урT3 & $30(49.2)$ & $27(62.8)$ & \\
\hline урT4 & $4(6.6)$ & $1(2.3)$ & \\
\hline \multicolumn{4}{|l|}{ Pathologic N stage } \\
\hline ypN0 & $50(82.0)$ & $34(79.1)$ & 0.802 \\
\hline ypN+ & $11(18.0)$ & $9(20.9)$ & \\
\hline \multicolumn{4}{|l|}{$\begin{array}{l}\text { Tumor regression } \\
\text { grade(TRG) }\end{array}$} \\
\hline$\leq 2$ & $16(26.2)$ & $17(39.5)$ & 0.344 \\
\hline 3 & $33(54.1)$ & $17(39.5)$ & \\
\hline 4 & $12(19.7)$ & $9(20.9)$ & \\
\hline
\end{tabular}

Data are given as $\mathrm{n}(\%)$ or mean (range).

abnormalities in patients after neoadjuvant radiotherapy $[15,16]$. The current study reinforces that forceps biopsy is of limited clinical value due to the inherent flaws of inability to obtain specimens deep to the muscularis or subserosa layer.

To date, there is no study reporting the value of core needle biopsy in predicting a pCR. Therefore, we hypothesized that core needle biopsy might improve the diagnostic performance. In the current study, we first confirmed the hypothesis by performing an ex vivo core needle biopsy in the tumor bed in the resected specimen. Our results showed that the overall accuracy was significantly improved compared to forceps biopsy $(76.7 \%$ vs. $36.1 \% ; p<0.001)$. We further tested the hypothesis by in vivo core needle biopsy guided by ERUS in good responders. Although the method is able to identify additional $36.4 \%$ of patients with residual disease after ruling out patients with obvious non$\mathrm{pCR}$, the overall accuracy is far from perfect. These were not unexpected since a false-negative result was 
Table 2: Demographics of patients underwent in vivo core needle biopsy

\begin{tabular}{|c|c|}
\hline Demographics & In vivo core needle biopsy $(n=16)$ \\
\hline Age, y & $58.5(40-82)$ \\
\hline Gender (female/male) & $8 / 8$ \\
\hline Distance from anal verge, $\mathrm{cm}$ & $4.3(2.0-8.0)$ \\
\hline Interval between RT and biopsy, days & $44.7(29-67)$ \\
\hline Interval between RT and surgery, days & $56.5(35-69)$ \\
\hline \multicolumn{2}{|l|}{ Clinical T stage } \\
\hline $\mathrm{cT} 2$ & $1(6.3)$ \\
\hline cT3 & $8(50.0)$ \\
\hline cT4 & $7(43.8)$ \\
\hline \multicolumn{2}{|l|}{ Clinical N stage } \\
\hline $\mathrm{cN} 0$ & $3(18.8)$ \\
\hline $\mathrm{cN}+$ & $13(81.3)$ \\
\hline \multicolumn{2}{|l|}{ Pathologic T stage } \\
\hline урT0 & $5(31.3)$ \\
\hline урT2 & $2(12.5)$ \\
\hline урT3 & $5(31.3)$ \\
\hline урT4 & $4(25)$ \\
\hline \multicolumn{2}{|l|}{ Pathologic N stage } \\
\hline ypN0 & $12(75)$ \\
\hline ypN+ & $4(25)$ \\
\hline \multicolumn{2}{|l|}{ Tumor regression grade (TRG) } \\
\hline$\leq 2$ & $4(25.0)$ \\
\hline 3 & $7(43.8)$ \\
\hline 4 & $5(31.3)$ \\
\hline
\end{tabular}

Data are given as $\mathrm{n}(\%)$ or mean (range).

Table 3: The result of histopathological findings of forceps biopsy compared with the surgical specimen

\begin{tabular}{l|c|c|c|}
\hline \multicolumn{2}{c}{ Forceps biopsy } & \multicolumn{2}{c}{ Surgical specimen } \\
\cline { 2 - 4 } \multicolumn{1}{c|}{ No-pCR } & Total \\
\hline Tumor $(+)$ & 10 & 0 & 10 \\
\hline Tumor $(-)$ & 39 & 12 & 51 \\
\hline Total & 49 & 12 & 61 \\
\hline
\end{tabular}

Sensitivity $=20.4 \%$; Specificity $=100 \%$; Positive predictive value $=100 \%$; negative predictive value $=23.5 \%$; accuracy $=36.1 \%$

more likely to occur in patients with minimal residual cancer cells or good responders, whom the in vivo core needle biopsy aims to. Moreover, we found a correlation between tumor response and sensitivity of ex vivo core needle biopsy, and the sensitivity was significantly higher in patients with mild or moderate response $(\mathrm{TRG} \leq 2)$ than patients with better response $(\mathrm{TRG}=3)$. Fewer patients were $\mathrm{TRG} \leq 2$ in the group of in vivo core needle biopsy, which may contribute to the low accuracy. Meanwhile, it might be more difficult to determine the target lesion in ERUS than direct observation in ex vivo core needle biopsy. 
Table 4: Correlation between TRG and forceps biopsy findings

\begin{tabular}{|l|c|c|c|}
\cline { 2 - 3 } \multicolumn{1}{c}{ TRG } & \multicolumn{2}{c}{ Torceps biopsy } & p value \\
\hline TRG $\leq 2$ & 5 & 11 & 0.351 \\
\hline TRG $=3$ & 5 & 28 & \\
\hline
\end{tabular}

TRG: tumor regression grade

Table 5: The result of histopathological findings of ex vivo core needle biopsy compared with the surgical specimen

\begin{tabular}{l|c|c|c|}
\hline \multirow{2}{*}{ Ex vivo core needle biopsy } & \multicolumn{2}{c}{ Surgical specimen } & \multirow{2}{*}{ Total } \\
\cline { 2 - 4 } \multicolumn{2}{c|}{ No-pCR } & 0 & 25 \\
\hline Tumor $(+)$ & 25 & 8 & 18 \\
\hline Tumor $(-)$ & 10 & 8 & 43 \\
\hline Total & 35 & \multicolumn{1}{c}{ pCR } \\
\hline
\end{tabular}

Sensitivity $=71.4 \%$; Specificity $=100 \%$; Positive predictive value $=100 \%$; negative predictive value $=44.4 \%$; accuracy $=76.7 \%$

Table 6: Correlation between TRG and ex vivo core needle biopsy

\begin{tabular}{|c|c|c|c|}
\hline \multirow[t]{2}{*}{ TRG } & \multicolumn{2}{|c|}{ ex vivo core needle biopsy } & \multirow{2}{*}{$p$ value } \\
\hline & Tumor $(+)$ & Tumor (-) & \\
\hline $\mathrm{TRG} \leq 2$ & 16 & 1 & 0.017 \\
\hline $\mathrm{TRG}=3$ & 9 & 8 & \\
\hline
\end{tabular}

TRG: tumor regression grade

Table 7: The result of histopathological findings of in vivo core needle biopsy compared with the surgical specimen

\begin{tabular}{|l|c|c|c|}
\hline \multirow{2}{*}{ In vivo core needle biopsy } & \multicolumn{2}{c}{ Surgical specimen } & Total \\
\cline { 2 - 4 } \multicolumn{1}{c|}{ No-pCR } & 0 & 4 \\
\hline Tumor $(+)$ & 4 & 5 & 12 \\
\hline Tumor $(-)$ & 7 & 5 & 16 \\
\hline Total & 11 & \multicolumn{2}{c}{ pCR } \\
\hline
\end{tabular}

Sensitivity $=36.4 \%$; Specificity $=100 \%$; Positive predictive value $=100 \%$; negative predictive value $=41.7 \%$; accuracy $=56.3 \%$

Of note, one patient (11.1\%) obtained ex vivo core needle biopsy achieved primary tumor regression (ypT0) but had residual tumor cells in the lymph nodes (ypN+). This incidence ranged from $2 \%$ to $17 \%$ in previous studies $[17,18]$. The inability of those biopsy approaches or radical imagine techniques to accurately identify these patients might challenge the less aggressive strategy.

Limitations in this study should be mentioned. Several studies demonstrated that a prolonged interval to assess tumor response could maximize tumor regression and result in a higher chance of pCR $[19,20]$. In this study, patients underwent ex vivo core needle biopsy nearly 2 weeks later than other approaches, which may account for some discrepancies. In addition, this study was performed in a single-institution and the selected population of in vivo core needle biopsy in good responders might also leaded to bias.

In conclusion, the current study showed that forceps biopsy was of limited clinical value in identifying patients with pCR after CRT. Although, core needle biopsy might further identify a subset of patients with residual cancer cells, the accuracy was not substantially increased in good responders. 


\section{MATERIALS AND METHODS}

\section{Patients}

Between Nov. 2011 and Dec. 2013, a total of 120 patients with LARC treated with nCRT at Sun Yat-sen University Cancer Center were prospectively included in this study. This study was approved by the Institutional Ethical Review Boards of the Sun Yat-sen University Cancer Center, and performed in accordance with the ethical standards laid down in the Declaration of Helsinki. Written informed consent was obtained from each patient before enrollment in the trial. All patients were biopsyproven adenocarcinoma. Clinical staging of local tumor was performed by Magnetic Resonance Image (MRI) and endoscopic ultrasound (EUS).

The total dosage of radiotherapy was 50 Gy consisted of 23 fractions of 2 Gy to clinical target volume and 2 fractions of 2 Gy to gross tumor volume. Patients received two cycles of modified XELOX (Oxaliplatin $100 \mathrm{mg} / \mathrm{m}^{2} \mathrm{~d} 1$, Capecitabine $1000 \mathrm{mg} / \mathrm{m}^{2}$ bid, $\mathrm{d} 1-14$ ) regimens on day 1-14 and day 21-34 concomitant to radiotherapy. The standard TME surgery was planned 6 to 8 weeks after completion of nCRT.

\section{Forceps biopsy, ex vivo core needle biopsy, and in vivo core needle biopsy}

Firstly, 61 patients were consecutively enrolled into the forceps biopsy group to evaluate the value in prediction of $\mathrm{pCR}$. These patients underwent forceps biopsies during the endoscopic examination using 2.8$\mathrm{mm}$ biopsy forceps about 6 weeks after the completion of nCRT. At least 3 biopsy samples were taken from the suspicious sites from every patient.

To evaluate the possible role of core needle biopsy in the diagnosis of $\mathrm{pCR}, 43$ patients were subsequently enrolled to the ex vivo core needle biopsy group before proceeding with the procedure in vivo. The standard TME surgery was performed 6 to 8 weeks after the end of nCRT. Once the specimen was removed and opened, the researcher performed an ex vivo core needle biopsy with an 18-gauge core needle direct to three representative regions of the tumor bed. In patients with suspected macroscopic complete response, the scar tissue was biopsied accordingly.

To validate the ex vivo findings and evaluate the safety of the procedure, core needle biopsy was further performed in vivo about 6 weeks after the completion of nCRT in 16 patients with good response. The definition of good response, determined by clinical examination, endoscopy and forceps biopsy, and MRI, was (1) substantial tumor downsizing ( $>30 \%$ reduction of the initial tumor size); (2) No obvious residual tumor or deep ulceration at endoscopy. A superficial ulcer or scar might be acceptable; and (3) negative forceps biopsies from the ulcer or former tumor location. Before the biopsy, patients received routine cleansing enema by $0.1 \%$ soap solution. Patients were examined initially with the ultrasound probe in the left lateral decubitus position. When hypoechoic area of the rectal wall was identified through ERUS and felt to represent the suspicious site of residual tumor, core needle biopsy would be performed under direct ultrasound guidance with an 18 -gauge core needle. At least 3 samples were achieved in each patient.

\section{Pathologic assessment}

All the biopsy samples were sent for routine pathological analysis and compared with the histopathological result of the resected specimens. Sensitivity, specificity, positive predictive value, negative predictive value, and accuracy were analyzed.

Pathological stage was determined according to the AJCC 7th edition [21]. Neoadjuvant CRT effect was evaluated using tumor regression grade (TRG) system by Dworak et al as follows [22]: Grade 0: no regression; Grade 1: dominant tumor mass with obvious fibrosis and/ or vasculopathy; Grade 2: dominantly fibrotic changes with few tumor cells or groups (easy to find); Grade 3 (or nearly pCR): very few (difficult to find microscopically) tumor cells in fibrotic tissue with or without mucous substance; Grade 4: no tumor cells, only fibrotic mass (total regression or response).

\section{Statistical analysis}

Statistical analysis was performed using the chisquare and Fisher's exact tests. A value of $p<0.05$ was considered statistically significant. Statistical Package for the Social Sciences (SPSS)' version 16.0 for Windows (SPSS, Inc. Chicago, IL) was used for all analysis.

\section{CONFLICTS OF INTEREST}

The authors declare no conflict of interest.

\section{GRANT SUPPORT}

This study was supported by the National Natural Science Foundation of China (81101591), and Natural Science Foundation of Guangdong Province, China (2011040005278, 9151008901000157).

\section{REFERENCES}

1. Sauer R, Becker H, Hohenberger W, Rödel C, Wittekind C, Fietkau R, Martus P, Tschmelitsch J, Hager E, Hess CF, Karstens JH, Liersch T, Schmidberger H, et al. Preoperative versus postoperative chemoradiotherapy for rectal cancer. N Engl J Med. 2004; 351:1731-40. 
2. Maas M, Nelemans PJ, Valentini V, Das P, Rödel C, Kuo LJ, Calvo FA, García-Aguilar J, Glynne-Jones R, Haustermans K, Mohiuddin M, Pucciarelli S, Small W Jr, et al. Long-term outcome in patients with a pathological complete response after chemoradiation for rectal cancer: a pooled analysis of individual patient data. Lancet Oncol. 2010; 11:835-44.

3. Gao YH, Zhang X, An X, Cai MY, Zeng ZF, Chen G, Kong LH, Lin JZ, Wan DS, Pan ZZ, Ding PR. Oxaliplatin and Capecitabine concomitant with neoadjuvant radiotherapy and extended to the resting period in high risk locally advanced rectal cancer. Strahlenther Onkol. 2014; 190:158-64.

4. Maas M, Beets-Tan RG, Lambregts DM, Lammering G, Nelemans PJ, Engelen SM, van Dam RM, Jansen RL, Sosef M, Leijtens JW, Hulsewé KW, Buijsen J, Beets GL. Wait-and-see policy for clinical complete responders after chemoradiation for rectal cancer. J Clin Oncol. 2011; 29:4633-40.

5. Habr-Gama A, Perez RO, Nadalin W, Sabbaga J, Ribeiro U Jr, Silva e Sousa AH Jr, Campos FG, Kiss DR, GamaRodrigues J. Operative versus nonoperative treatment for stage 0 distal rectal cancer following chemoradiation therapy: long-term results. Ann Surg. 2004; 240:711-7.

6. Habr-Gama A, Perez RO, Wynn G, Marks J, Kessler H, Gama-Rodrigues J. Complete clinical response after neoadjuvant chemoradiation therapy for distal rectal cancer: characterization of clinical and endoscopic findings for standardization. Dis Colon Rectum. 2010; 53:1692-8.

7. Guillem JG, Chessin DB, Shia J, Moore HG, Mazumdar M, Bernard B, Paty PB, Saltz L, Minsky BD, Weiser MR, Temple LK, Cohen AM, Wong WD. Clinical examination following preoperative chemoradiation for rectal cancer is not a reliable surrogate end point. J Clin Oncol. 2005; 23:3475-9.

8. Glynne-Jones R, Wallace M, Livingstone JIL, MeyrickThomas J. Complete clinical response after preoperative chemoradiation in rectal cancer: Is a "Wait and See" policy justified?. Dis Colon Rectum. 2008; 51:10-9.

9. Hiotis SP, Weber SM, Cohen AM, Minsky BD, Paty PB, Guillem JG, Wagman R, Saltz LB, Wong WD. Assessing the predictive value of clinical complete response to neoadjuvant therapy for rectal cancer: an analysis of 488 patients. J Am Coll Surg. 2002; 194:131-5.

10. Perez RO, Habr-Gama A, Gama-Rodrigues J, Proscurshim I, Julião GP, Lynn P, Ono CR, Campos FG, Silva e Sousa AH Jr, Imperiale AR, Nahas SC, Buchpiguel CA. Accuracy of positron emission tomography/computed tomography and clinical assessment in the detection of complete rectal tumor regression after neoadjuvant chemoradiation LongTerm Results of a Prospective Trial (National Clinical Trial 00254683). Cancer. 2012; 118:3501-11.

11. Kuo LJ, Chiou JF, Tai CJ, Chang CC, Kung CH, Lin SE, Hung CS, Wang W, Tam KW, Lee HC, Liang HH, Chang YJ, Wei PL. Can we predict pathologic complete response before surgery for locally advanced rectal cancer treated with preoperative chemoradiation therapy? Int J Colorectal Dis. 2012; 27:613-21.

12. Meterissian S, Skibber J, Rich T, Roubein L, Ajani J, Cleary K, Ota DM. Patterns of residual disease after preoperative chemoradiation in ultrasound $\mathrm{T} 3$ rectal carcinoma. Ann Surg Oncol. 1994; 1:111-6.

13. Perez RO, Habr-Gama A, Pereira GV, Lynn PB, Alves PA, Proscurshim I, Rawet V, Gama-Rodrigues J. Role of biopsies in patients with residual rectal cancer following neoadjuvant chemoradiation after downsizing: can they rule out persisting cancer? Colorectal Dis. 2012; 14:714-20.

14. Duldulao MP, Lee W, Streja L, Chu P, Li W, Chen Z, Kim J, Garcia-Aguilar J. Distribution of residual cancer cells in the bowel wall after neoadjuvant chemoradiation in patients with rectal cancer. Dis Colon Rectum. 2013; 56:142-9.

15. Hayden DM, Jakate S, Pinzon MC, Giusto D, Francescatti AB, Brand MI, Saclarides TJ. Tumor scatter after neoadjuvant therapy for rectal cancer: are we dealing with an invisible margin?. Dis Colon Rectum. 2012; 55:1206-12.

16. Smith FM, Wiland H, Mace A, Pai RK, Kalady MF. Depth and Lateral Spread of Microscopic Residual Rectal Cancer after Neoadjuvant Chemoradiation: Implications for Treatment Decisions. Colorectal Dis. 2014; 16:610-5.

17. Pucciarelli S, Capirci C, Emanuele U, Toppan P, Friso ML, Pennelli GM, Crepaldi G, Pasetto L, Nitti D, Lise M. Relationship between pathologic T-stage and nodal metastasis after preoperative chemoradiotherapy for locally advanced rectal cancer. Ann Surg Oncol. 2005; 12:111-6.

18. Hughes R, Glynne-Jones R, Grainger J, Richman P, Makris A, Harrison M, Ashford R, Harrison RA, Livingstone JI, McDonald PJ, Meyrick Thomas J, Mitchell IC, Northover JM, et al. Can pathological complete response in the primary tumor following pre-operative pelvic chemoradiotherapy for T3-T4 rectal cancer predict for sterilization of pelvic lymph nodes, a low risk of local recurrence and the appropriateness of local excision? Int $\mathrm{J}$ Colorectal Dis. 2006; 21:11-7.

19. Sloothaak DA, Geijsen DE, van Leersum NJ, Punt CJ, Buskens CJ, Bemelman WA, Tanis PJ. Optimal time interval between neoadjuvant chemoradiotherapy and surgery for rectal cancer. Br J Surg. 2013; 100:933-9.

20. Moore HG, Gittleman AE, Minsky BD, Wong D, Paty PB, Weiser M, Temple L, Saltz L, Shia J, Guillem JG. Rate of pathologic complete response with increased interval between preoperative combined modality therapy and rectal cancer resection. Dis Colon Rectum. 2004; 47:279-86.

21. Edge SB, Compton CC. The American Joint Committee on Cancer: the 7th edition of the AJCC cancer staging manual and the future of TNM. Ann Surg Oncol. 2010; 17:1471-4.

22. Dworak O, Keilholz L, Hoffmann A. Pathological features of rectal cancer after preoperative radiochemotherapy. Int J Colorectal Dis. 1997; 12:19-23. 